\title{
ON CONVOLUTIONS WITH THE MÖBIUS FUNCTION
}

\author{
S. L. SEGAL
}

ABSTRACT. By using the results of [6], it is proved that for an extensive class of increasing functions $h$,

$$
\sum_{1 \leqq d \leqq x} \frac{\mu(d)}{d} h\left(\frac{x}{d}\right) \sim x h^{\prime}(x) \text { as } x \rightarrow \infty
$$

where $\mu$ denotes the Möbius function. This result incidentally settles affirmatively Remark (iii) of [6], and refines the Tauberian Theorem 2 of that paper. It is also shown that one type of condition imposed in [6] is necessary to the truth of the cited Theorem 2 , at least if some sort of quasi-Riemann hypothesis is true. Nevertheless, examples are given to show that on the one hand $\left(^{*}\right)$ may be true for functions not covered by the first theorem of this paper, and on the other that some sort of nonnaïve condition on a function $h$ is necessary to ensure the truth of $\left({ }^{*}\right)$.

Much of this note, as will be evident, is in the nature of an interesting addendum to [6]; had I had the wit to notice it earlier, it should of course have been incorporated there.

Throughout, $x$ denotes a real variable and $s=\sigma+i t$ a complex variable, $\sigma, t$ real. All sums begin at 1 . Given a suitable function $g, \mathscr{I}$ will denote the operator defined by $\mathscr{I} g(y)=\int_{1}^{y}(g(x) / x) d x$, and $\mathscr{I} r$ the $r$ th iterate of $\mathscr{I}$. $\mu$ is the Möbius function and $N(x)=\sum_{n \leqq x}(\mu(n) / n) . \zeta(s)$ is the Riemann zeta-function, $\gamma$ is Euler's constant. When $h(x)$ is constant, the right side of $(*)$ is to be interpreted as equal to $o(1)$.

For convenience we state here the main results of [6] which will be used in the sequel:

THEOREM A. Let $f(x)$ be any function bounded and integrable in every finite subinterval of $[1, \infty)$ which satisfies

$$
\sum_{v \leqq x} f\left(\frac{x}{v}\right)=x g(x)+o\left(x^{2} g^{\prime}(x)\right)
$$

where $g(x)$ is a positive, twice continuously differentiable function, defined

Received by the editors August 11, 1971.

AMS 1970 subject classifications. Primary 10H25; Secondary 40E05, $10 \mathrm{H} 05$.

Key words and phrases. Möbius function, Dirichlet convolution, Tauberian theorems, Riemann zeta-function, Ingham summability.

(c) American Mathematical Society 1972 
on $[1, \infty)$ such that

(i) $g^{\prime}(x)>0$ for $x \in(1, \infty)$,

(ii) $x g^{\prime}(x)$ is nonincreasing from some point on,

(iii) for some positive integer $k, x(\log x)^{k} g^{\prime}(x)=u(x)$ is nondecreasing from some point on, and $\lim \inf _{x \rightarrow \infty} u(x)=\infty$.

Then

as $x \rightarrow \infty$.

$$
\int_{1}^{x} \frac{f(t)}{t^{2}} d t=g(x)-\gamma \sum_{v \leqq x} \frac{\mu(v)}{v} g\left(\frac{x}{v}\right)+o\left(x g^{\prime}(x)\right)
$$

(This is Theorem 2 of [6].)

THEOREM B. If $f$ and $g$ are as in Theorem $\mathrm{A}$, then

$$
\int_{1}^{x} \frac{f(t)}{t^{2} g^{\prime}(t)} d t=x+o(x) .
$$

(This is equation (19) of [6]; if $f$ is nondecreasing, one then easily deduces $f(x) \sim x^{2} g^{\prime}(x)$ as in Theorem 1 of [6].)

We now state

THEOREM 1. Let $h(x)$ be a positive function which has the property that there exists a nonnegative integer $r$ such that $h \in C^{r+2}(1, \infty)$ and $h(x)=$ Ir $g(x)$ where g satisfies (i), (ii), (iii) of Theorem A; then

$$
\sum_{d \leqq x} \frac{\mu(d)}{d} h\left(\frac{x}{d}\right) \sim x h^{\prime}(x) \text { as } x \rightarrow \infty .
$$

Proof. By induction. For convenience, we define $g(x)=0$ for $x<1$. Suppose first the theorem were true for some $k, k \geqq 1$. Let $G(x)=\mathscr{I}^{k} g(x)$. Then (since $k \geqq 1$ ) $G$ is clearly unbounded, and by hypothesis

and so

$$
\sum_{d \leqq x} \frac{\mu(d)}{d} G\left(\frac{x}{d}\right) \sim x G^{\prime}(x) \text { as } x \rightarrow \infty,
$$

$$
\begin{aligned}
\sum_{d \leqq x} \frac{\mu(d)}{d} \int_{1}^{x / d} \frac{G(t)}{t} d t & =\int_{1}^{x} \frac{1}{t} \sum_{d \leqq t} \frac{\mu(d)}{d} G\left(\frac{t}{d}\right) d t \\
& =\int_{1}^{x} G^{\prime}(t)(1+o(1)) d t=G(x)+o(G(x)),
\end{aligned}
$$

which establishes the theorem for $r=k+1$. It remains to prove the theorem for $r=0$ and $r=1$. For $r=0, h(x)=\mathscr{I}^{0} g(x)=g(x)$. Let

$$
F(x)=\sum_{d \leqq x} \frac{\mu(d)}{d} g\left(\frac{x}{d}\right) .
$$


Then

and so, by Theorem A,

$$
\sum_{d \leqq x} \frac{x}{d} F\left(\frac{x}{d}\right)=x g(x)
$$

$$
\int_{1}^{x} \frac{F(t)}{t} d t=g(x)-\gamma \sum_{v \leqq x} \frac{\mu(v)}{v} g\left(\frac{x}{v}\right)+o\left(x g^{\prime}(x)\right) ;
$$

and, by Theorem B,

$$
\int_{1}^{x} \frac{F(t)}{\operatorname{tg}^{\prime}(t)} d t=x+o(x) .
$$

(If $x F(x)$ were monotone, the conclusion, as remarked previously, would now follow almost immediately; unfortunately this need not be the case.)

Furthermore the hypotheses on $g$ imply (see Lemma 1 of [6] for the easy deduction)

$$
\begin{aligned}
\lim _{x \rightarrow \infty} \frac{x g^{\prime \prime}(x)}{g^{\prime}(x)} & =-1 \text { and also that } \\
\frac{x g^{\prime \prime}(x)}{g^{\prime}(x)} & =\frac{(d / d x)\left(x g^{\prime}(x)\right)}{g^{\prime}(x)}-1 \leqq-1,
\end{aligned}
$$

from some point on. Hence $x g^{\prime \prime}(x) / g^{\prime}(x)$ is eventually nondecreasing and so eventually has a nonnegative derivative, a.e.

Now, integrating by parts in (5), after using (4), gives

$$
\begin{aligned}
\int_{1}^{x} \frac{F(t)}{\operatorname{tg}^{\prime}(t)} d t & =\frac{1}{g^{\prime}(x)} \int_{1}^{x} \frac{F(t)}{t} d t+\int_{1}^{x} \frac{g^{\prime \prime}(t)}{\left(g^{\prime}(t)\right)^{2}} \int_{1}^{t} \frac{F(u)}{u} d u d t \\
& =\frac{g(x)}{g^{\prime}(x)}-\frac{\gamma F(x)}{g^{\prime}(x)}+o(x)+\int_{1}^{x} \frac{g^{\prime \prime}(t)}{\left(g^{\prime}(t)\right)^{2}}\left(g(t)-\gamma F(t)+o\left(\operatorname{tg}^{\prime}(t)\right)\right) d t \\
& =x-\frac{\gamma F(x)}{g^{\prime}(x)}-\gamma \int_{1}^{x} \frac{F(t) g^{\prime \prime}(t)}{\left(g^{\prime}(t)\right)^{2}} d t+o(x)
\end{aligned}
$$

(cf. treatment of equation (15) in [6]).

And so (5) yields

$$
\frac{F(x)}{g^{\prime}(x)}=-\int_{1}^{x} \frac{F(t) g^{\prime \prime}(t)}{\left(g^{\prime}(t)\right)^{2}} d t+o(x) .
$$

But

$$
\begin{aligned}
\int_{1}^{x} \frac{F(t) g^{\prime \prime}(t)}{\left(g^{\prime}(t)\right)^{2}} d t & =\int_{1}^{x} \frac{F(t)}{t g^{\prime}(t)} \frac{\operatorname{tg}^{\prime \prime}(t)}{g^{\prime}(t)} d t \\
& =\frac{x g^{\prime \prime}(x)}{g^{\prime}(x)} \int_{1}^{x} \frac{F(t)}{\operatorname{tg}^{\prime}(t)} d t-\int_{1}^{x} \frac{d}{d t}\left(\frac{t g^{\prime \prime}(t)}{g^{\prime}(t)}\right) \int_{1}^{t} \frac{F(u)}{u g^{\prime}(u)} d u d t+O(1),
\end{aligned}
$$


and on substituting this in (8), and using (6) and (5), we get

$$
\begin{aligned}
\frac{F(x)}{g^{\prime}(x)} & =x+o(x)+\int_{1}^{x} \frac{d}{d t}\left(\frac{\operatorname{tg}^{\prime \prime}(t)}{g^{\prime}(t)}\right)(t+o(t)) d t \\
& =x+o(x)+o\left(\int_{1}^{x} t d\left(\frac{t g^{\prime \prime}(t)}{g^{\prime}(t)}\right)\right) .
\end{aligned}
$$

However, by (6),

$$
\begin{aligned}
\int_{1}^{x} t d\left(\frac{t g^{\prime \prime}(t)}{g^{\prime}(t)}\right) & =\frac{x^{2} g^{\prime \prime}(x)}{g^{\prime}(x)}-\int_{1}^{x} \frac{\operatorname{tg}^{\prime \prime}(t)}{g^{\prime}(t)} d t+O(1) \\
& =-x+o(x)+\int_{1}^{x}(1+o(1)) d t+O(1)=o(x),
\end{aligned}
$$

and substituting this in (9) gives

$$
F(x) \sim x g^{\prime}(x)
$$

as claimed. This proves the case $r=0$. The case $r=1$ now follows on substituting (10) in (4) to obtain

$$
\int_{1}^{x} \frac{F(t)}{t} d t=g(x)-\gamma x g^{\prime}(x)+o\left(x g^{\prime}(x)\right),
$$

and noting that

$$
\sum_{d \leqq x} \frac{\mu(d)}{d} \int_{1}^{x / d} \frac{g(t)}{t} d t=\int_{1}^{x} \frac{F(t)}{t} d t,
$$

and that the conditions on $g$ imply $x g^{\prime}(x)=o(g(x))$ as $x \rightarrow \infty$.

REMARKS. (a) To prove the results of [6] used above, it was necessary to invoke a fairly strong form of the prime number theorem: $N(x)=$ $o\left((\log x)^{-k}\right)$ for every $k>0$. In the other direction, for $k$ a positive integer, $(\log x)^{k}$ satisfies the conditions placed on $h(x)$ in Theorem 1 (with $g(x)=\log x)$, and so we get, from Theorem 1 ,

$$
\sum_{d \leqq x} \frac{\mu(d)}{d}\left(\log \frac{x}{d}\right)^{k} \sim k(\log x)^{k-1}
$$

for every positive integer $k$ (which is, of course, also deducible directly from $N(x)=o\left((\log x)^{-k}\right)$ for every $\left.k>0\right)$.

(b) The conclusion of Theorem A can now be amended to read:

$$
\int_{1}^{x} \frac{f(t)}{t^{2}} d t=g(x)-\gamma x g^{\prime}(x)+o\left(x g^{\prime}(x)\right)
$$

$g(x)$ was assumed for this theorem to be twice continuously differentiable. 
In practice, however, $g$ is usually analytic. Suppose we assume $g$ is analytic; under what subsidiary conditions on $g$ can (12) be replaced by a similar asymptotic expansion with a desired number of terms for

$$
\int_{1}^{x}\left(F(t) / t^{2}\right) d t ?
$$

Condition (iii) of Theorem A seems somewhat unnatural; while it may be somewhat ameliorated by using stronger results from prime number theory than used in [6] (see Remark (iv) of that paper), the real question is whether any condition in addition to (i) and (ii) is necessary for the truth of Theorem A. Assuming a "quasi-Riemann hypothesis", the answer is "yes", as Theorem 2 below shows.

THEOREM 2. Suppose some sort of quasi-Riemann hypothesis holds; i.e. suppose $\zeta(s) \neq 0$ for some strip $b<\sigma \leqq 1\left(b \geqq \frac{1}{2}\right)$. Then there is a function $k(x)$ satisfying (i) and (ii) of Theorem A which is analytic in $(1, \infty)$ and for which the conclusion of Theorem A (in the form of (12)) is false. A similar contradiction is even easier to obtain in Theorem A's original form.

Proof. Let $a \in(b, 1)$. Define $k_{a}(x)=1-x^{a-1}$. Then $k_{a}(x)$ satisfies (i), (ii) as is easily checked. By the hypothesis on $\zeta$ we have that, as $x \rightarrow \infty$,

$$
\int_{1}^{x} \frac{N(t)}{t} d t=-1+o\left(x^{a-1}\right)
$$

and

$$
\int_{1}^{x} \frac{N(t)}{t^{a}} d t \text { converges to } \frac{1}{(a-1) \zeta(a)} .
$$

(These results arise from using partial summation on

$$
\sum_{d \leqq x} \frac{\mu(d) \log d}{d} \text { and } \sum_{d \leqq x} \frac{\mu(d)}{d^{a}}=\sum_{d \leqq x} \frac{\mu(d)}{d} d^{1-a},
$$

and taking note of results usually stated in the literature for $b=\frac{1}{2}$ (the Riemann hypothesis), but whose analogues for other values of $b$ are immediate, see e.g. [5], [7, p. 315].)

$$
\text { Let } \begin{aligned}
f(x)= & (1-a) x^{a} \int_{1}^{x}\left(N(t) / t^{a}\right) d t \text {. Then } \\
\sum_{d \leqq x} f\left(\frac{x}{d}\right) & =(1-a) x^{a} \sum_{d \leqq x} \frac{1}{d^{a}} \int_{1}^{x / d} \frac{N(t)}{t^{a}} d t \\
& =(1-a) x^{a} \int_{1}^{x} u^{-a} \sum_{d \leqq u} \frac{1}{d} N\left(\frac{u}{d}\right) d u=x\left(1-x^{a-1}\right)=x k_{a}(x),
\end{aligned}
$$


since $\sum_{d \leqq u}(1 / d) N(u / d)=1$, and $N(x)=0$ for $x<1$. If the conclusion expressed by (12) held for all $k_{a}(x)$, then we would have

$$
\begin{aligned}
\int_{1}^{x} \frac{f(t)}{t^{2}} d t & =(1-a) \int_{1}^{x} t^{a-2} \int_{1}^{t} \frac{N(u)}{u^{a}} d u d t \\
& =1-x^{a-1}-\gamma(1-a) x^{a-1}+o\left(x^{a-1}\right) \text { for all } a, b<a<1
\end{aligned}
$$

But integrating by parts in (15) gives

$$
-x^{a-1} \int_{1}^{x} \frac{N(u)}{u^{a}} d u-\int_{1}^{x} \frac{N(t)}{t} d t=1-(1+\gamma(1-a)) x^{a-1}+o\left(x^{a-1}\right)
$$

and so by (13), as $x \rightarrow \infty$,

$$
\int_{1}^{x} \frac{N(u)}{u^{a}} d u=1+\gamma(1-a)+o(1) \text { for all } a, \frac{1}{2} \leqq b<a<1 ;
$$

or by (14),

$$
\frac{1}{(a-1) \zeta(a)}=1+\gamma(1-a) \text { for all } a, \frac{1}{2} \leqq b<a<1 .
$$

For a given fixed value of $a$, (16) might be shown false by ad hoc computation, however, this would not suffice to prove the theorem for any quasi-Riemann hypothesis; instead, we simply argue as follows: (16) would imply

$$
\frac{\zeta(a)-(1 /(a-1))-\gamma}{a-1}=\frac{\gamma^{2}}{1-\gamma(a-1)}, \text { for all } a, \frac{1}{2} \leqq b<a<1 .
$$

Letting $a \rightarrow 1$ in (17) would give

$$
\lim _{a \rightarrow 1^{-}} \frac{\zeta(a)-(1 /(a-1))-\gamma}{a-1}=\gamma^{2} .
$$

But the limit on the left is known to equal

$$
-\gamma_{1} \stackrel{\text { def }}{=}-\lim _{n \rightarrow \infty}\left(\sum_{v=1}^{n} \frac{\log v}{v}-\frac{1}{2}(\log n)^{2}\right)=0.07281+\neq \gamma^{2}
$$

which is the desired contradiction. (Two proofs of the form taken by the coefficients of the Taylor expansion of $\zeta(s)-1 /(s-1)$ about 1 may be found in [1]; the result was known to Hardy in 1912, and is no doubt much older still. The computation of $-\gamma_{1}$ was made by Wilton [8] from a different expression for that constant.) 
We close with two examples further illuminating the relationship $\left({ }^{*}\right)$.

EXAMPLE 1. There is a nondecreasing function $h$ for which (ii) does not hold but $\left(^{*}\right)$ still does.

Proof. Take $h(x)=\int_{1}^{x}((1+N(t)) / t) d t$. It is easy to see that $|N(x)| \leqq 1$ for all $x \geqq 1$ [4, p. 583] whence $h$ is nondecreasing; clearly $x h^{\prime}(x)=$ $1+N(x)$ is not monotone decreasing. However,

$$
\begin{aligned}
\sum_{d \leqq x} \frac{\mu(d)}{d} & h\left(\frac{x}{d}\right) \\
& =\sum_{d \leqq x} \frac{\mu(d)}{d} \log \left(\frac{x}{d}\right)+\sum_{d \leqq x} \frac{\mu(d)}{d} \int_{1}^{x / d} \frac{N(t)}{t} d t \\
& =1+o(1)-\sum_{d \leqq x} \frac{\mu(d)}{d}\left(\sum_{m \leqq x / d} \frac{\mu(m) \log m}{m}-\sum_{m \leqq x / d} \frac{\mu(m)}{m} \log \left(\frac{x}{d}\right)\right) \\
& =1+o(1)-2 \sum_{d \leqq x} \frac{\mu(d)}{d} \sum_{m \leqq x / d} \frac{\mu(m) \log m}{m}+\log x \sum_{d \leqq x} \frac{\mu(d)}{d} \sum_{m \leqq x / d} \frac{\mu(m)}{m} \\
& =1+o(1),
\end{aligned}
$$

since $\sum_{d \leqq x}(\mu(d) \log d / d)=-1+o(1)$ (e.g. $\left.[4, \S 158]\right)$,

$$
\sum_{d \leqq x} \frac{\mu(d)}{d} \sum_{m \leqq x / d} \frac{\mu(m) \log m}{m}=o(1), \quad \text { and } \quad \sum_{d \leqq x} \frac{\mu(d)}{d} \sum_{m \leqq x / d} \frac{\mu(m)}{m}=o\left(\frac{1}{\log x}\right)
$$

as $x \rightarrow \infty$; these last two being easily proved by a technique going back to Dirichlet and embodied in the argument in [4, p. 685].

EXAMPLE 2. There is a function $h$ such that $h(x) \sim C$ as $x \rightarrow \infty, C$ a constant, but $\sum_{d \leqq x}(\mu(d) / d) h(x / d) \neq o(1)$ as $x \rightarrow \infty$.

Proof. It is known that there are $(I)$-summable series which are not convergent; i.e. there is a sequence $\left\{a_{n}\right\}$ such that $\lim _{x \rightarrow \infty}(1 / x) \sum_{n \leqq x} \sum_{d \mid n} d a_{d}$ exists but $\sum a_{n}$ does not converge. (This is stated in [3]; a proof may be obtained by applying the Silverman-Toeplitz Theorem to the inverse of the (I)-transformation.) It is also known that $(I)$-summability implies $(C, 1)$-summability [3]. Let

$$
I(x)=\frac{1}{x} \sum_{n \leqq x} \sum_{d \mid n} d a_{d}=\frac{1}{x} \sum_{d \leqq x} \sum_{m \leqq x / d} m a_{m}
$$

where $\left\{a_{n}\right\}$ is such a sequence. Then

$$
\sum_{d \leqq x} \frac{\mu(d)}{d} I\left(\frac{x}{d}\right)=\frac{1}{x} \sum_{m \leqq x} m a_{m}
$$


$\lim _{x \rightarrow \infty} I(x)$ exists, $\sum a_{n}$ is $(C, 1)$-summable but not convergent, and so $\sum_{m \leqq x} m a_{m} \neq o(x)$ as $x \rightarrow \infty$ (e.g. [2, Theorem 65]).

REMARK. No explicit example of such a sequence $\left\{a_{n}\right\}$ seems to be known.

\section{REFERENCES}

1. W. E. Briggs and S. Chowla, The power series coefficients of $\zeta(s)$, Amer. Math. Monthly 62 (1955), 323-325. MR 16, 999.

2. G. H. Hardy, Divergent series, Clarendon Press, Oxford, 1949. MR 11, 25.

3. A. E. Ingham, Some Tauberian theorems connected with the prime number theorem, J. London Math. Soc. 20 (1945), 171-180. MR 8, 147.

4. E. Landau, Handbuch der Lehre von der Verteilung der Primzahlen, 2nd ed., Chelsea, New York, 1953. MR 16, 904.

5. J. E. Littlewood, Quelques conséquences de l'hypothèse que la fonction $\zeta(s)$ de Riemann n'a pas de zéros dans le demi-plan $R(s)>\frac{1}{2}$, Comptes Rendus 154 (1912), 263266.

6. S. L. Segal, A general Tauberian theorem of Landau-Ingham type, Math. Z. 111 (1969), 159-167. MR 40 \#2624.

7. E. C. Titchmarsh, The theory of the Riemann zeta-function, 2nd ed., Clarendon Press, Oxford, 1951. MR 13, 741.

8. J. R. Wilton, $A$ note on the coefficients of the expansion of $\zeta(s, x)$ in powers of $s-1$, Quart. J. Math. 50 (1927), 329-332.

Department of Mathematics, University of Rochester, Rochester, New YORK 14627 